\title{
A BUNCH KILLER FOR THE NSLS X-RAY ELECTRON STORAGE RING*
}

\author{
R.J. Nawrocky, U. Bergmann, and D.P. Siddons \\ National Synchrotron Light Source, Brookhaven National Laboratory, Upton NY $119.3 \mathrm{~F}$ \\ JUL 271993
}

Abstract

In the NSLS $x$-ray electron storage ring, which operates at a harmonic number of 30 , the beam may be stored in many different bunch patterns. The minimum spacing between bunches is approximately 19 nsec. While most of the experimenters are primarily interested in photon flux, some experiments are sensitive to bunch spacing. Time resolved nuclear resonance scattering experiments, for example, need pulses of $x$-rays spaced of the order of 100 nsec apart and a very low noise floor $\left(10^{-6}\right)$ between pulses. Perhaps even more important than the level of the background is that it be reproducible and homogeneous in time. It has been found in practice that a small number of electrons always get trapped in the "empty" of buckets during injection into the storage ring and remain as low level stray bunches. These extra bunches produce an unacceptable temporally localized, nonreproducible background which is difficult if not impossible to correct for. A "bunch killer" system based on the if knockout tochnique has been developed and installed on the ring to remove the unwanted bunches. We describe the operation of this system and present experimental results to illustrate its effectiveness.

\section{INTRODUCTION}

The NSLS $x$-ray storage ring operates with an accelerating of frequency $f_{r f}=52.88 \mathrm{MHz}$ and a harmonic number $n=30$. The ring energy is ramped after injection from $750 \mathrm{MeV}$ up to approximately $2.5 \mathrm{GeV}$. The machine is usually filled with 25 consecutive bunches. Some fraction of the machine operation is devoted to special fill patterns, usually single or five bunch modes. This provides the capability for performing time-resolved experiments in the nanosecond range. For example, nuclear resonance scattering experiments [1] need to maintain a very low background (a factor of $10^{-6}$ ) between consecutive light pulses spaced some 100 nsec or more apart.

In practice we find that some elextrons get trapped in the "empty" if buckets during injection which in effect raises the background. This noise, which is time-localized and typically different from fill to fill, is difficult to correct for. It should be noted that at the present time the very low level stray bunches can only be observed by the synchrotron radiation that they produce using photon counting $x$-ray detectors after the beam has been stored and ramped up in energy. The low level stray bunches can not be readily seen with conventional if beam pick-ups or visible light monitors.

* Work performed under the auspices of the U.S. DOE.
After a preliminary investigation, $\mathrm{O} v \mathrm{~B}$ comchuded that removing the unwanted bunches after filling would lequire less effort than cleaning up the injection process itself.

The low background requirement prompted the development of a "bunch killer" system. This system uses a stripline kicker to induce large coherent vertical oscillations in the unwanted bunches at the betatron frequency [2] until they collide with the vacuum chamber wall and are lost. The kicker is driven by a gated, swept if signal which is synchronized with the stored bunch pattern. The if amplifiers driving the kicker are limited in output power, so that the stray bunches can only be knocked out at low energy. The kickers are driven sinusoidally at a single frequency within a betatron sideband which is only a few $\mathrm{kHz}$ wide. Because this frequency can vary from fill to fill by as much as $\pm 175 \mathrm{kHz}$ ( \pm 0.05 tune units), the frequency of the excitation is modulated back and forth across its nominal value (see Fig.1). In the $x$-ray ring, the fractional part of the vertical tune, $q$, is nominally equal to 0.36 and the available stripline is best coupled to the beam in the $100 \mathrm{MHz}$ range. As a result, the kicker is driven at the frequency of the upper betatron sideband of the 60th revolution harmonic $\left(60 \mathrm{f}_{\mathrm{o}}\right)$ which is 106.4 MHz.

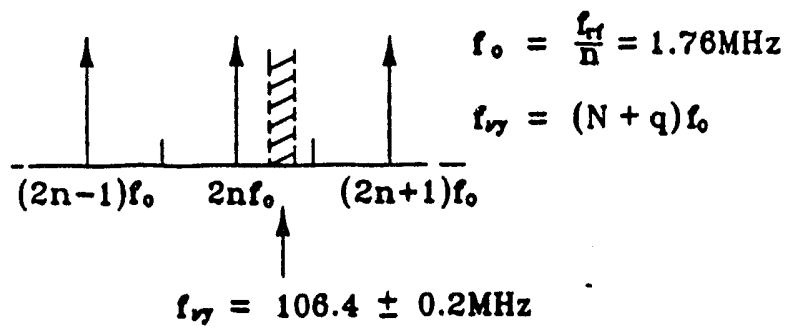

Fig. 1. Vertical betatron frequency during injection.

The bunch killer system bas been installed and used in routine operations; photon counting data indicate that the system is effective in removing stray bunches to a level where they can no longer be distinguished from the random background noise. The system requires a minimum of intervention from the machine operator and is now being upgraded for completely automatic operation .

\section{SYSTEM DESCRIPTION}

A block diagram of the system is shown in Fig. 2. The kicker drive signal from a $106.4 \mathrm{MHz}$ VCO is gated on and off by means of a double balanced mixer. The IF input of 
CONTROL ROOM

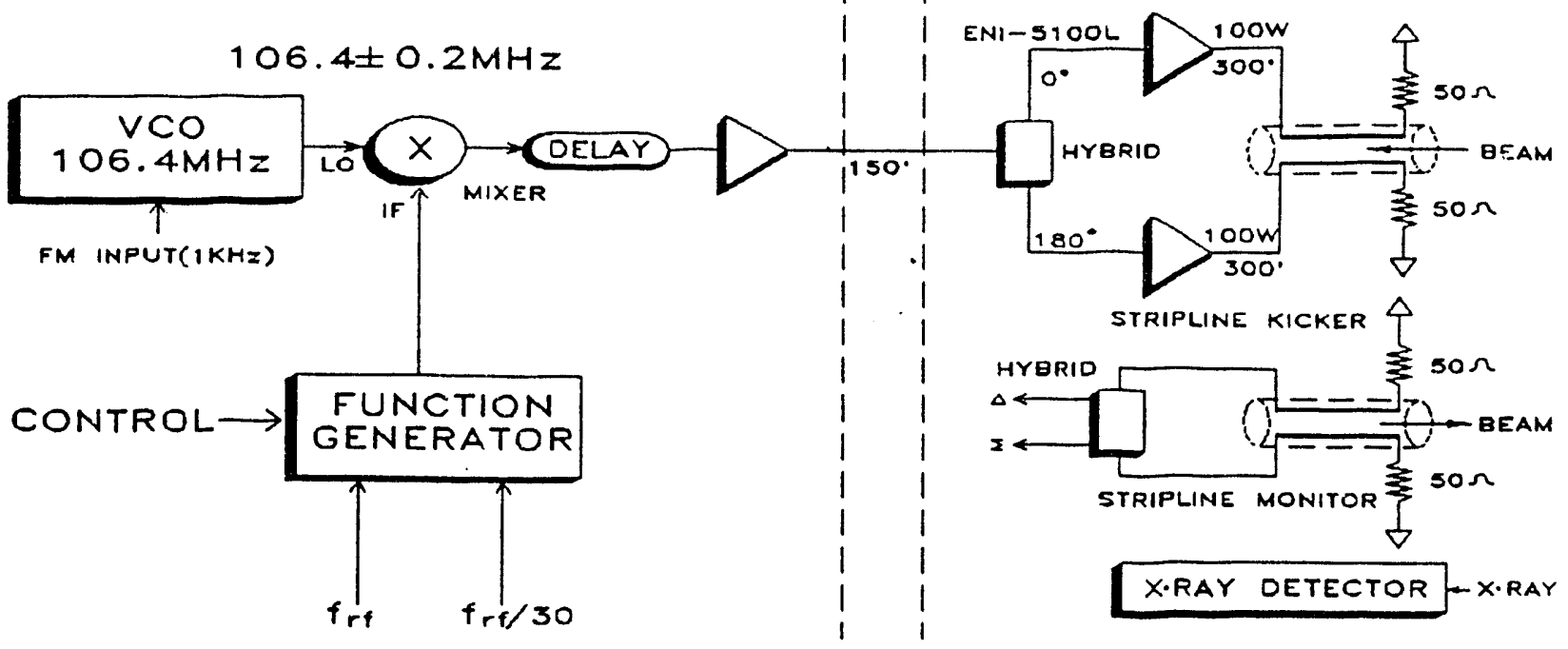

Fig. 2. System block diagram̀

the mixer is driven by a programmable function generator (PFG). The PFG, which also provides a bias to the mixer input for clean gating of the VCO signal, is synchronized with signals at $f_{r f}$ and $f_{\text {rff }} / 30$. The PFG has several modes of operation tailored to the various bunch patterns of interest which can be selected by the computer control system. The mixer output is split and amplified by two 100 Watt if power amplifiers (ENI Model 5100L) to drive the stripline differentially. The gated drive and a sum signal proportional to beam intensity derived from another of stripline are monitored with a fast digital oscilloscope. The kicker is driven at $106.4 \mathrm{MHz}$ because it is best coupled to the beam in this frequency range.

As mentioned earlier, the tune of the machine may vary from fill to fill by as much as \pm 0.05 units. The resonance line, however, is narrow (a few $\mathrm{kHz}$ ), and the amplifiers driving the kicker are limited in power. This means that we must drive with a single frequency within the betatron sideband to excite the resonance. To simplify th: process of finding the correct frequency during operations, we modulate the output of the $106.4 \mathrm{MHz}$ oscillator by \pm 200 $\mathbf{k H z}$ at $1 \mathrm{kHz}$ rate. The transverse oscillations are induced in the beam by sweeping back and forth through the resonance. The excitation of these oscillations by the kicker is counteracted to a small degree by radiation damping. Since the radiation damping time constant at low energy is of the order of two hundred msec, the excitation is sufficiently strong to build up the amplitude of the oscillations and drive the stray particles into the vacuum chamber wall.

To demonstrate the operation of the bunch killer, we first generated an artificially "bad" five bunch fill and then removed the unwanted bunches by turning the system on, as shown in Fig.3. The lower trace of Fig.3a shows the bunch pattern with small intermediate bunches as observed with the if stripline monitor. The upper trace in the same figure is the gated if drive which is to be applied to the kicker electrodes. Since there are arbitrary delays introduced in various signal paths, it is necessary to compensate for them by trial and error until the correct phase relationship between the drive and the bunch pattern is found. With the correct delay established and the drive signal connected to the kicker, the intermediate bunches are reduced to a point where they can no longer be observed with the rf pick-up. This is shown in the lower trace of Fig.3b. Since the bunch spacing is only $19 \mathrm{nsec}$, the jitter

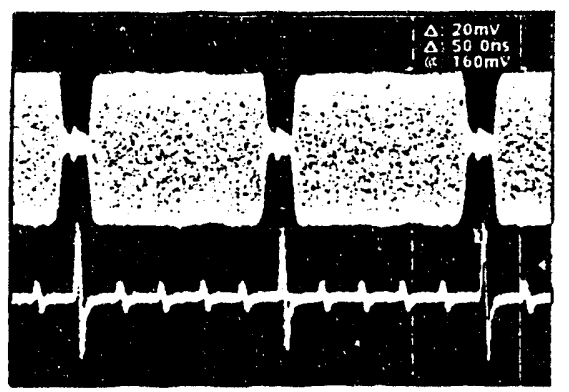

a.

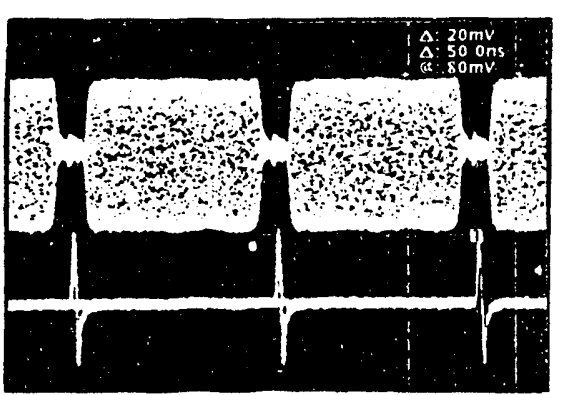

b.

Fig. 3. Mixer output and beam pick-up signal with kicker off (a), and with kicker on (b). 
between the kicker excitation and the beam pattern must be controlled to a few nsec so as not to affect the desired bunches. This has been readily achieved in the NSLS system as can be seen in Fig. 3 where the waveforms are the envelopes of many oscilloscope traces.

\section{EXPERIMENTAL RESULTS}

As previously mentioned, the level of the unwanted bunches is typically too low to be detected by the if pick-up detectors. The best technique so far is to use the set-up for nuclear resonance experiments as a diagnostic. This uses a fast plastic scintillator detector which is usually employed to probe the decay of nuclei excited by synchrotron radiation. A block diagram of the detector is shown in Fig.4. An x-ray emitted by a stray bunch and registered by the detector starts a time-to-analog converter (TAC). A legitimate beam pulse signal (the prompt) derived from a fast photo-diode looking at synchrotron light stops the TAC. The TAC output, which is proportional to the time delay between the two events, is digitized and stored in the computer memory. The process is repeated for a large number of events and the distribution is plotted as a function of the delay. Results for a five bunch fill with and without the bunch killer are shown in Fig.5. Fig. 5a shows the presence of stray bunches at $19 \mathrm{nsec}$ and at $38 \mathrm{nsec}$ after the prompt. The level of these bunches is roughly 100 times the expected signal level for the nuclear resonance experiment. With the bunch killer turned on, stray bunches can not be distinguished from the background (Fig. Sb).

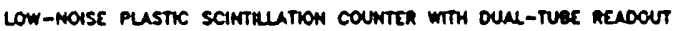

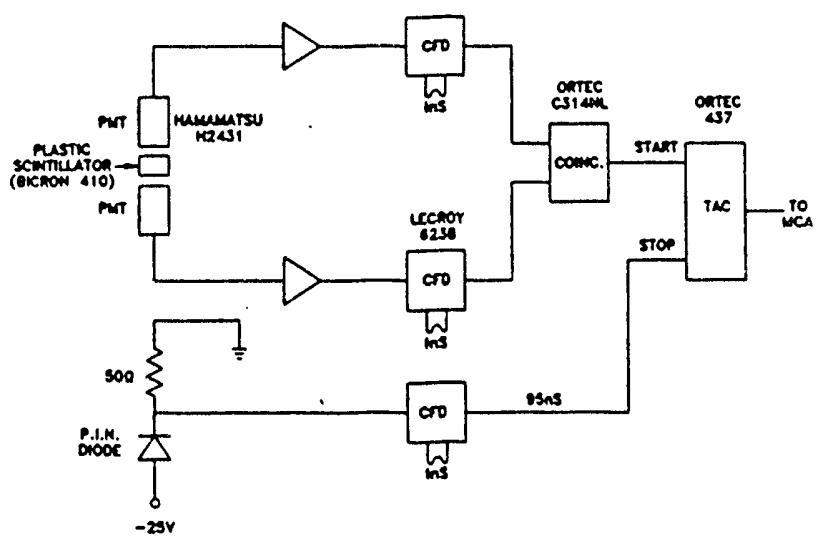

Fig. 4. The $x$-ray detector.

\section{ACKNOWLEDGEMENTS}

The function generator used in the NSLS bunch killer was designed and constructed by J. Tallent. The authors would like to thank Dr. E. Blum for reviewing the manuscript.
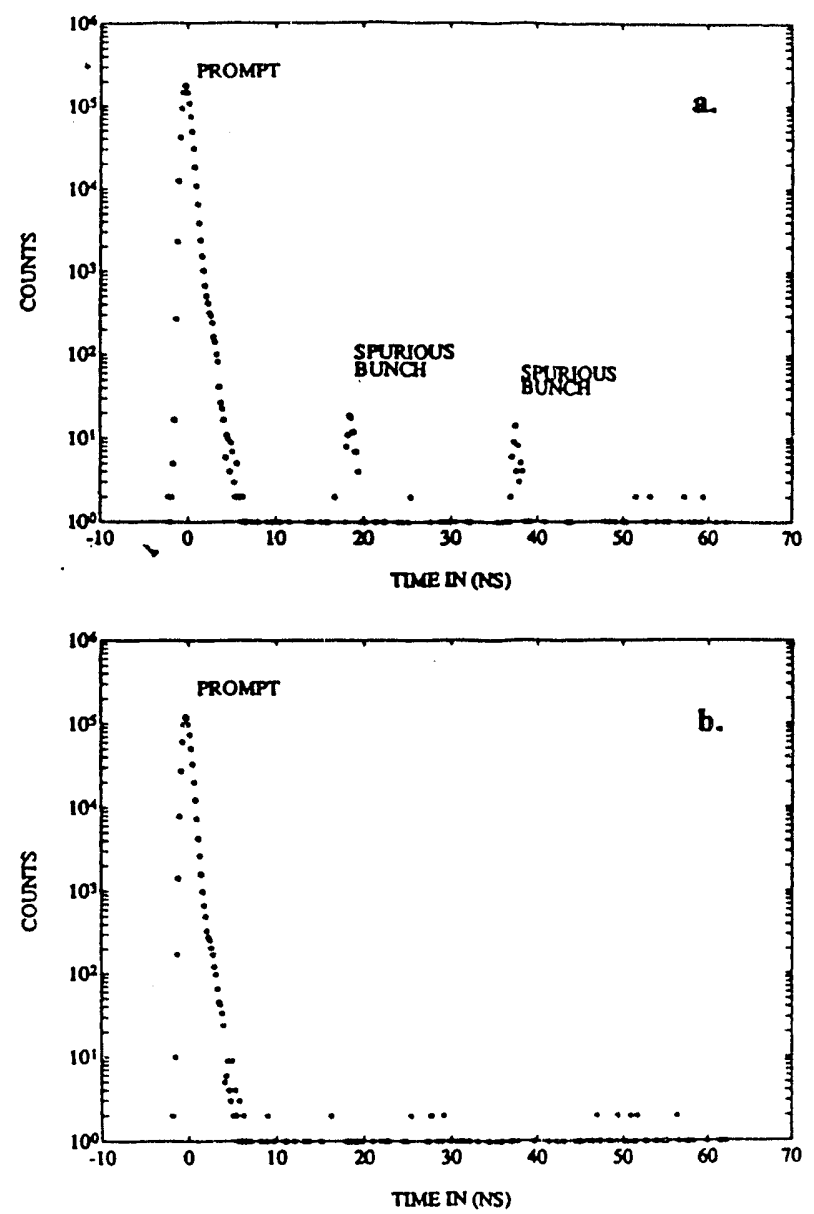

Fig. 5. Typical detector output with bunch killer off (a), and with bunch killer on (b).

\section{REFERENCES}

U. van Buerck, et al., "Nuclear forward scattering of synchrotron radiation", Physical Review B, Vol.22, No.10, 6207 (1992).

[2] E. Wilson, "Transverse beam dynamics", in Proceedings of CAS, P. Bryant and S. Tumer ed., CERN 85-19, Vol.1, 64 (1985).

\section{DISCLAIMER}

This report was prepared as an account of work sponsored by an agency of the United States Government. Neither the United States Government nor any agency thereof, nor any of their employees, makes any warranty, express or implied, or assumes any legal liability or responsibility for the accuracy, completeness, or usefulness of any information, apparatus, product, or process disclosed, or represents that its use would not infringe privately owned rights. Reference herein to any specific commercial product, process, or service by trade name, trademark, ence herein to any sectionmendation, or favoring by the United States Government or any agency thereof. The views and opinions of authors expressed herein do not necessarily state or reflect those of the United States Government or any agency thereof. 

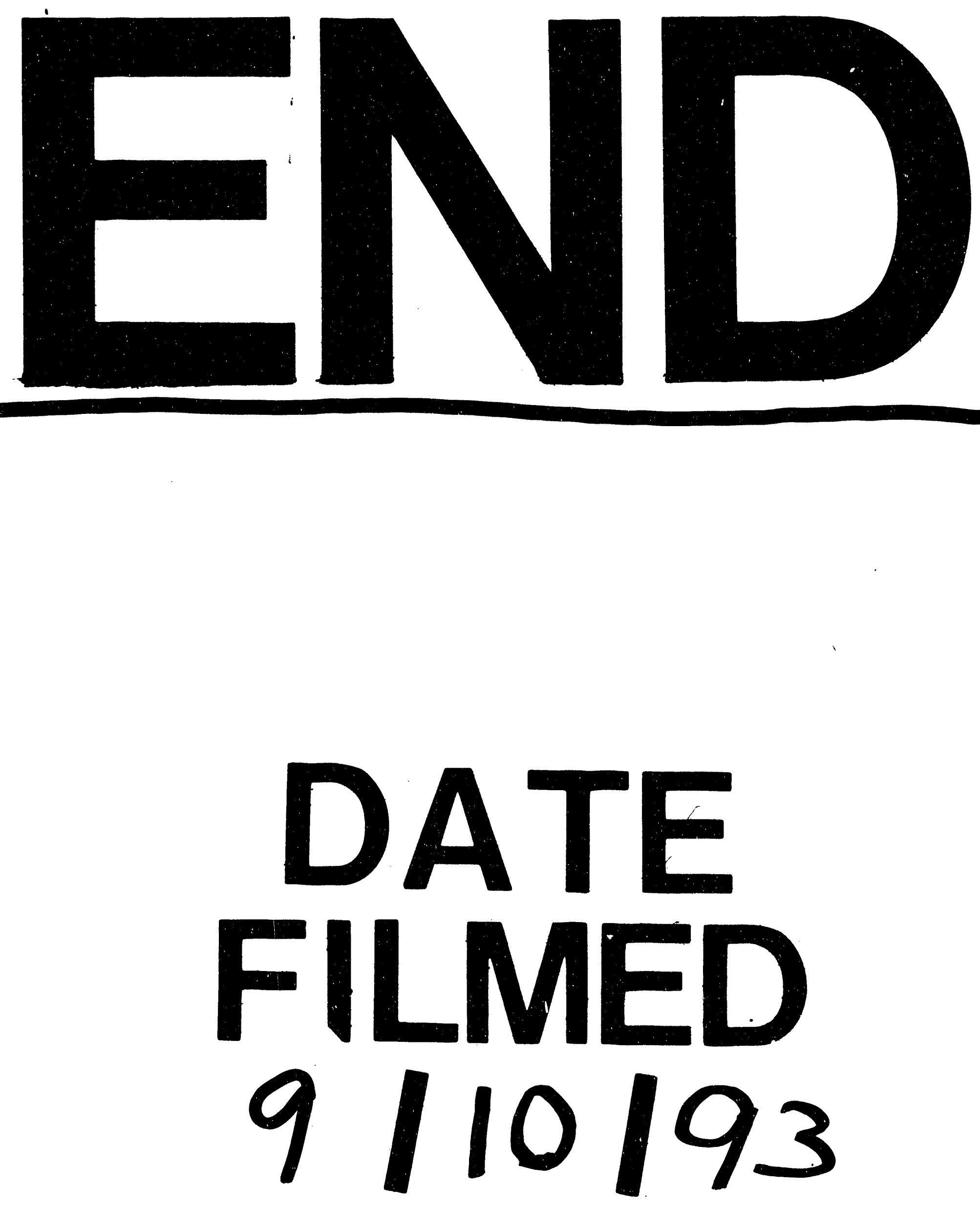
